\title{
Evaluación de la eventual presencia de agroquímicos en distintos sustratos en una arrocera del Chaco Húmedo
}

\author{
Falcón, F. Natalia; Currie, H. María; Michellod, Miguel \\ Cátedra de Hidrología Agrícola. Facultad de Ciencias Agrarias - Universidad Nacional del Nordeste. \\ Sargento Cabral No 2131 (3400) Corrientes, Argentina. TE/FAX (03794) 427589/ 427131- Interno 129 \\ f.nataliafalcon@gmail.com
}

\begin{abstract}
Resumen
Los defensivos agrícolas son herramientas indispensables de la agricultura moderna por su eficacia para limitar los efectos perjudiciales ocasionados por plagas y enfermedades disminuyendo el valor productivo de los cultivos. Los objetivos del presente trabajo fueron establecer la eventual presencia de agroquímicos en muestras de agua, sedimento, suelo y granos de arroz y la posible incidencia de la posición relativa y la fecha de muestreo. El seguimiento se efectuó en las arroceras San Carlos-Cancha Larga, provincia del Chaco, extrayéndose 156 muestras en las campañas 2010/2011 y 2011/2012, en distintas etapas del ciclo del cultivo. Las muestras, fueron remitidas al laboratorio QUINEA, para determinar la eventual presencia de agroquímicos; la metodología consistió en tratamientos de muestras con recuperación de metanol a través de columnas SPE-C18 y análisis de extractos: utilizando Cromatografía Líquida (HPLC) y Cromatografía Gaseosa. Los agroquímicos analizados fueron: Bentazón; Bispyribac sódico; Clamazone; Glifosato; Imazapic+Imazapir; Pendimetalin; Profoxidim; Picloram; Cyhalofop Butil Fipronil; Gamma+LambdaCialotrina; Metamidofos. Aquellos que sobrepasen el Límite Detectable ( $\mathrm{LD}=0.002 \mathrm{ppm})$, se comparan con los Límites Máximos Permisibles (LMP ppm). Para agua de consumo los LMP fueron extraídos de la Secretaria de Recursos Naturales y Ambiente Humano, Decreto 831/93; Ex - SAGPyA: Resolución 507/2008 y del SENASA: 256/2003 y 934/2010. Para alimentos los LMP fueron tomados de la Organización de las Naciones Unidas para la Agricultura y la Alimentación (FAO/OMS), CODEX ALIMENTARIUS. Es posible afirmar que ningún principio activo de los agroquímicos analizados sobrepasan los LMP; las fechas de muestreos durante un ciclo de riego, no muestran diferencias significativas entre sí, y no se registran ingresos ni egresos de agroquímicos al sistema de riego.
\end{abstract}

Palabras clave: Análisis, permanencia, defensivos agrícolas.

\section{INTRODUCCIÓN Y ANTECEDENTES}

El agroecosistema arrocero se comporta como un humedal artificial temporario, alternando periodos de inundación en verano y de sequía en invierno, otorgándole al sistema arrocero una heterogeneidad espacial y temporal que facilita el establecimiento de elementos de la biodiversidad de humedales (Ramsar $\mathrm{xx} 31$ ).

El arroz (Oryza spp) es el principal cultivo a nivel mundial, cubriendo alrededor del $11 \%$ de las tierras arables del planeta (Fasola y Ruiz 1996).

El arroz constituye un alimento estratégico en la composición de la dieta de más de la mitad de la población mundial, y junto al trigo y la soja representan uno de los principales commodities producidos. $\mathrm{La}$ mayor parte de la producción mundial es consumida en la región de origen, en cambio, Argentina, es un "exportador neto", localizándose su producción en cinco Provincias del Nordeste, Entre Ríos 42\%, Corrientes 35\%, Santa Fe 16\%, Chaco 3\% y Formosa 3\% (Moulin, et al., 2011).
Los defensivos agrícolas son una herramienta indispensables de la agricultura moderna por su eficacia para limitar los efectos perjudiciales de insectos, enfermedades y malezas en los cultivos. (Arregui y Puricelli, 2008).

En el caso de los fungicidas, su uso se ha intensificado en los últimos años en arroz irrigado debido a la mayor incidencia de enfermedades, de la misma forma el uso de insecticidas es consecuencia de diferentes tipos de plagas que comprometen la productividad del cultivo (Teló et al., 2011).

Los insecticidas organofosforado (OP) son compuestos típicamente miscibles en solventes orgánicos y excepcionalmente en agua; son los insecticidas más empleados en el mundo estimándose que representan el $34 \%$ del mercado mundial de este tipo de plaguicida, siendo un OP el Metamidofos. (Arregui y Puricelli, 2008).

Los piretroides, son productos poco selectivos sobre la fauna benéfica. Los piretroides sintéticos similares y modelados a partir de piretrinas y han 
evolucionado en cuatro generaciones, la cuarta generación esta en uso desde 1975 hasta la actualidad, es interesante debido a su eficacia en un rango de dosis muy bajas 0,1 a $0,01 \mathrm{~kg}$ i.a.ha ${ }^{-1}$. Esta generación incluye entre otros a Cipermetrina,Gammacialotrina, Lambdacialotrina, los cuales son fotoestables, presentan una volatilidad mínima, lo que implica una residualidad extendida de hasta 10 días en condiciones óptimas (Arregui y Puricelli, 2008).

Actualmente, los herbicidas son una parte integral de las modernas prácticas agrícolas. Proveen un método eficiente, flexible y barato de control de malezas, contribuyendo su acción a sustanciales incrementos en rendimientos y calidad de los cultivos (Arregui y Puricelli, 2008).

Los residuos de Glifosato que permanecen en los cultivos tratados, cuando es utilizado apropiadamente y la aplicación cumple con las buenas prácticas agrícolas, no generan efectos dañinos en los seres humanos o en los animales. (European Commission, 2002).

El objetivo de este trabajo fue establecer la eventual presencia de agroquímicos en distintos sustratos del sistema arrocero: agua, sedimento, suelo y granos de arroz, adicionalmente se analizó, la posible incidencia de la posición relativa, y la fecha de muestreo en los potenciales casos que la presencia de agroquímicos, resulta significativa.

\section{MATERIALES Y MÉTODOS}

El trabajo se realizó en las arroceras "San Carlos" y "Cancha Larga" ubicadas en la provincia del Chaco, en el departamento Bermejo, con las siguientes coordenadas de ubicación: Latitud Sur $27^{\circ}$ 4' 45,5'; Longitud Oeste $58^{\circ} 40^{\prime}$ '54,3”.

La toma de muestras se efectuó durante las campañas 2010/2011 y 2011/2012, obteniéndose 156 muestras, extraídas en distintas etapas del ciclo del cultivo de arroz (presiembra (PS); inicio riego (IR), pleno riego (PR), cosecha(C)). Los puntos de muestreos se encuentran en las siguientes coordenadas de ubicación, muestras de grano $\mathrm{N}^{\circ} 1 \mathrm{y} \mathrm{N}^{\circ} 2$, en silos situados en cercanías del Río Paraguay, en cuanto a las muestras de suelo, agua, y sedimento se detallan en las tablas $\mathrm{N}^{\mathrm{o}} 1 \mathrm{y} \mathrm{N}^{\mathrm{o}} 2$.

Tabla $\mathbf{N}^{0} \mathbf{1}$. Ubicación y coordenadas geográficas de los sitios de muestreos.

\begin{tabular}{lcc}
\hline \multicolumn{3}{c}{ Muestra de suelo } \\
\hline Muestras & Lat. Sur & Long. Oeste \\
\hline M № 1 & $27^{\circ} 04^{\prime} 52,6^{\prime \prime}$ & $58^{\circ} 40^{\prime} 44,9^{\prime \prime}$ \\
M No 2 & $27^{\circ} 05^{\prime} 50,4^{\prime \prime}$ & $58^{\circ} 40^{\prime} 50^{\prime \prime}$ \\
M № 3 & $27^{\circ} 05^{\prime} 23^{\prime \prime}$ & $58^{\circ} 45^{\prime} 51,3^{\prime \prime}$ \\
M № 4 & $27^{\circ} 06^{\prime} 45,8^{\prime \prime}$ & $58^{\circ} 45^{\prime} 09,5^{\prime \prime}$ \\
\hline
\end{tabular}

Tabla $\mathbf{N}^{\circ} 2$. Ubicación y coordenadas geográficas.

\begin{tabular}{cccl}
\hline & \multicolumn{3}{c}{ Muestras de agua y sedimento } \\
\hline Muestras & Latitud Sur & Longitud Oeste & Lugar de muestreo \\
\hline M № 1 & $27^{\circ} 26^{\prime} 56^{\prime \prime}$ & $58^{\circ} 38^{\prime} 35^{\prime \prime}$ & Río Paraguay \\
M № 2 & $27^{\circ} 05^{\prime} 45^{\prime \prime}$ & $58^{\circ} 39^{\prime} 15,2^{\prime \prime}$ & P. San Fernando \\
M № 3 & $27^{\circ} 4^{\prime} 04,5^{\prime \prime}$ & $58^{\circ} 41^{\prime} 30,3^{\prime \prime}$ & Lag. Moncholo \\
M № 4 & $27^{\circ} 57^{\prime} 50^{\prime \prime}$ & $58^{\circ} 40^{\prime} 55^{\prime \prime}$ & Canal \\
M № 5 & $27^{\circ} 05^{\prime} 08^{\prime \prime}$ & $58^{\circ} 47^{\prime} 53,3^{\prime \prime}$ & Arroyo Tatané \\
M № 6 & $27^{\circ} 05^{\prime} 08^{\prime \prime}$ & $58^{\circ} 47^{\prime} 53,3^{\prime \prime}$ & Canal de desagüe \\
M № 7 & $27^{\circ} 03^{\prime} 23,4^{\prime \prime}$ & $58^{\circ} 43^{\prime} 21,5^{\prime \prime}$ & Arroyo Caracol \\
\hline
\end{tabular}

En la tabla $\mathrm{N}^{0} 1$ se especifica la ubicación y coordenadas geográficas de las muestras de suelo, la muestra $\mathrm{N}^{\circ} 1$ fue extraída dentro de la chacra San Carlos, la muestra $\mathrm{N}^{\circ} 2$ fue tomada de la chacra nueva, de corta historia arrocera (menos de tres años), muestra $\mathrm{N}^{\circ} 3$ de la chacra vieja, con aptitud arrocera de más de 3 años y la última muestra, la $\mathrm{N}^{\circ} 4$ fue tomada dentro de la chacra Cancha Larga.

La tabla $\mathrm{N}^{\circ} 2$ puntualiza los sitios de muestreos de agua y sedimento. La muestra $\mathrm{N}^{\mathrm{o}} 1$, fue extraída de la toma del río Paraguay y, se constituye como un punto de ingreso al establecimiento San Carlos; las muestras $\mathrm{N}^{\circ} 2,3,4,5$ y 6 , fueron tomadas dentro de la chacra, plena actividad arrocera, y la última muestra la $\mathrm{N}^{\mathrm{o}} 7$, corresponde a la salida del agua del establecimiento Cancha Larga, específicamente aguas arribas del arroyo Caracol.

En cada muestreo se utilizaron planillas denominadas "Hoja de campo para muestreo de agua de uso agrícola", detallándose la ubicación y coordenadas geográficas, hora de inicio de cada muestreo, estado del tiempo, $\mathrm{pH}$, temperatura $\left(\mathrm{T}^{\circ}\right)$, conductividad eléctrica (C.E.), enumerado de fotos y un croquis del lugar.

Extraídas las muestras de agua son colocadas en conservadora a $5{ }^{\circ} \mathrm{C}$ y remitidas al Laboratorio de Análisis QUINEA. Los agroquímicos analizados son: Bentazón; Bispyribac sódico; Clamazone; Glifosato; Imazapic + Imazapir; Pendimetalin; Profoxidim; Picloram; Cyhalofop Butil e insecticidas Fipronil; Gamma + Lambdacialotrina; Metamidofós. El método empleado o spike se basa en el "Grado de Recuperación"; se trabaja con RP 18: C-18, un núcleo de Silicio con 18 átomos de carbono, siendo una molécula no polar.

Se parte de soluciones comerciales, no son métodos validados, son comunes y aptos.

Agregado de bicarbonato de sodio al 5\%, extracción y pre concentración en fase solida a través de columnas SPE-C18, con recuperación de Metanol, se recupera y se concentra la muestra, al recuperar el 90 y $105 \%$ es una extracción eficiente y satisfactoria y los análisis de extractos de agroquímicos: utilizando 
Cromatografía Líquida de Alta Resolución (HPLC) en fase reversa C18 con detección de UV y Cromatografía Gaseosa en detección FID y ECD. Comparación con estándares de referencia.

Obtenidos los resultados de laboratorio, se aplicó un Análisis Estadístico para establecer el grado de significancia si existiere entre los agroquímicos anteriormente nombrados. Aplicando el Test: Tukey con un error Alfa $=0.05$. En la tabla $\mathrm{N}^{\circ} 3$ se establecen los Límites Máximos Permisibles utilizados para cada agroquímico respectivamente: Secretaria de Recursos Naturales y Ambiente Humano, Decreto 831/93; Ex - SAGPyA: Resolución 507/2008, SENASA: 256/2003 y 934/2010 para agua de consumo y la Organización de las Naciones Unidas para la Agricultura y la Alimentación (FAO/OMS), Codex Alimentarius 2010; tomado como parámetro para establecer los Límites Máximos Permisibles (LMP) en alimentos. En la tabla $\mathrm{N}^{\mathrm{o}} 3$ se pone en evidencia la inexistencia de determinados productos en los distintos decretos nombrados anteriormente.

\section{RESULTADOS Y DISCUSIÓN}

Los resultados correspondientes a las muestras analizadas, no sobrepasaron los LMP establecidas por las normativas vigentes.

Diversos de los defensivos agrícolas no están presentes en la normativas citadas en la tabla $\mathrm{N}^{\mathrm{o}} 3$, en este caso se adoptó el LMP establecido por el Codex Alimentarius o se toma un valor por defecto de 0,01 mg. $\mathrm{kg}^{-1}$ correspondiente al Límite de Detección del método de análisis.
Se aplicaron pruebas estadísticas a los resultados obtenidos en los análisis de los defensivos agrícolas, de los cuales en ninguno de los casos tuvieron diferencias estadísticamente significativas y no pasaron el límite máximo permitido. Entre ellos se encuentra el Glifosato herbicida de contacto actualmente señalado (por tener efectos adversos sobre la salud humana animal y el ambiente), pero inexistentes en todas las muestras analizadas. De los agroquímicos restantes, se tuvieron en cuenta a modo de ejemplo para muestras de agua: Fipronil (curasemillas), Profoxidim (herbicida) y a través de un "Análisis Multivariado" se compararon los 12 agroquímicos. Para muestras de sedimento el Cyhalofop Butil (herbicida) y Gamma+LambdaCialotrina (insecticida) en los cuales se percibieron puntos claves de muestreos (dentro y fuera del área productiva).

En el gráfico $\mathrm{N}^{\mathrm{o}} 1$ se establecen diferencias entre las muestras $\mathrm{N}^{\circ} 1$ y $\mathrm{N}^{\circ} 2$ de granos, silos ubicados en cercanías del Río Paraguay, existiendo diferencias significativas entre muestras, pero ninguna sobrepasa el LMP. En la muestra 1, la mayoría de los agroquímicos presentaron las mayores concentraciones, si bien hay diferencia con la muestra 2, no supera los valores máximos establecidos de $0,05 \mathrm{mg}^{-1} \mathrm{l}^{-1}$.

Se expresan en el gráfico $\mathrm{N}^{\circ} 2$ las fechas de muestreos durante un ciclo de riego, las cuales no muestran diferencias reveladoras entre sí. No existen diferencias significativas entre fechas de muestreos y los diferentes agroquímicos no superan el límite máximo permitido.

Tabla No 3: Limites Máximos Permisibles para agua de consumo y alimento.

\begin{tabular}{|c|c|c|c|c|c|}
\hline Agroquímico & LD (ppm) & $\begin{array}{l}\text { Decreto } \\
831 / 93^{1}\end{array}$ & $\begin{array}{l}\text { Resolución } \\
256 / 2003^{1}\end{array}$ & 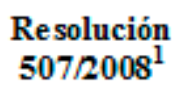 & $\begin{array}{l}\text { Resolución } \\
934 / 2010^{2}\end{array}$ \\
\hline Fipronil & 0,002 & $\mathrm{NE}$ & 0,01 & 0,01 & 0,01 \\
\hline G lifosato & 0,005 & 0,2 & 0,05 & 0,01 & 0,2 \\
\hline $\begin{array}{l}\text { G amma }{ }^{+} \\
\text {LambdaCialotrina }\end{array}$ & 0,002 & NE & 0,2 & NE & 0,2 \\
\hline Pendim etalin & 0,005 & NE & 0,05 & 0,05 & 0,05 \\
\hline Picloram & 0,005 & NE & 0,5 & 0,5 & 0,5 \\
\hline Cyhalofop Butil & 0,002 & NE & NE & NE & NE \\
\hline Metamidofos & 0,002 & NE & 0,05 & 0,05 & 0,05 \\
\hline Bispyribac sódico & 0,005 & NE & 0,05 & $\mathrm{NE}$ & 0,05 \\
\hline Imazapic+Imazapir & 0,005 & NE & 0,005 & 0,02 & 0,02 \\
\hline Profoxidim & 0,002 & NE & NE & NE & 0,05 \\
\hline Bentazón & 0,005 & NE & 0,05 & NE & 0,05 \\
\hline Clom asone & 0,002 & NE & 0,1 & NE & 0,1 \\
\hline
\end{tabular}

NE: no existe

${ }^{1:}$ Decreto para agua- ${ }^{2}$ Decreto para alimentos. 


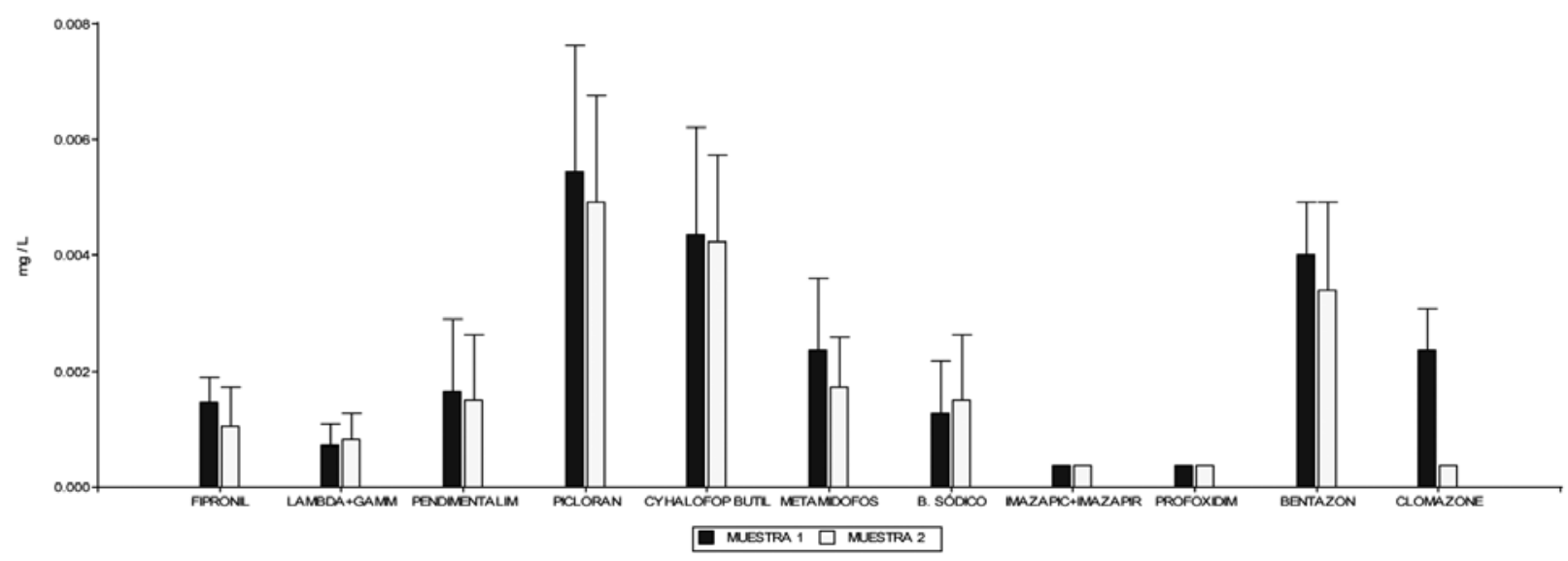

Gráfico $\mathbf{N}^{0}$ 1: Resultados de las muestras de granos.

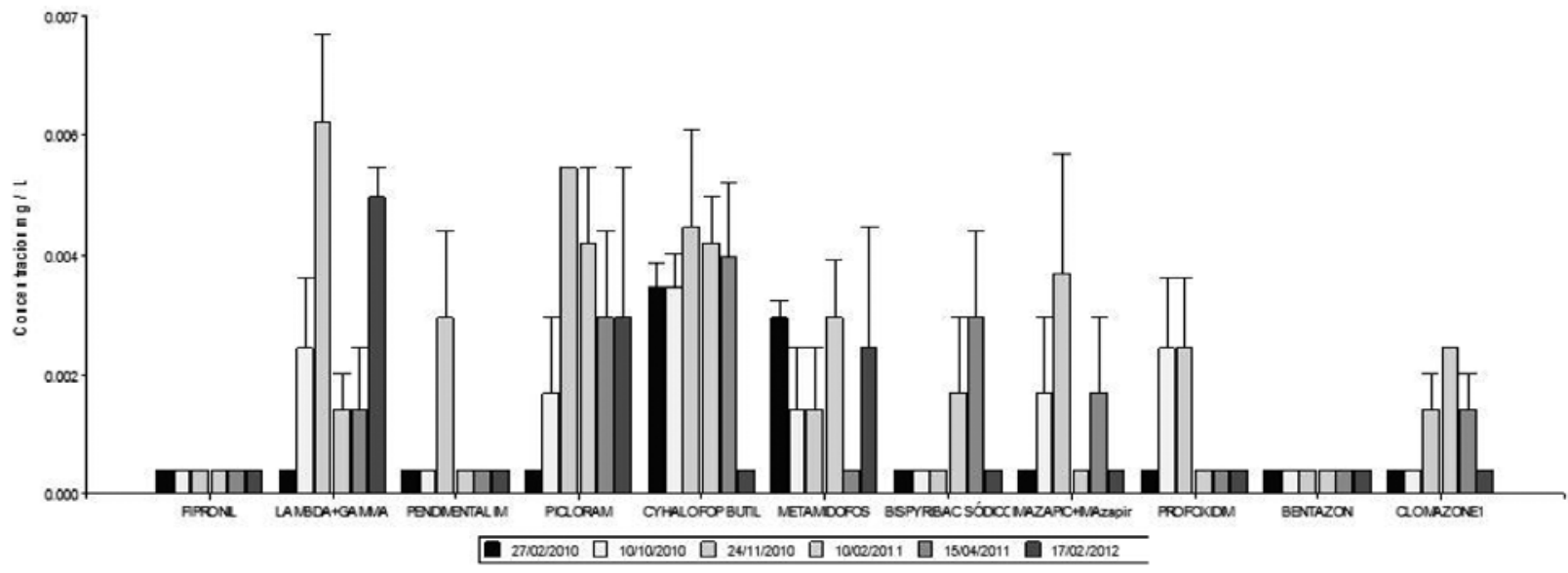

Gráfico $\mathbf{N}^{\mathbf{0}}$ 2: Resultado de las muestra de suelo.

Tabla N ${ }^{\circ} 4$. Muestra de agua: 1 (Toma del R. Paraguay), y 7 (tomadas fuera de la chacra), son significativamente diferentes a las muestras 4, 6, 5, 2 y 3 (tomadas dentro de la chacra) presentando estas últimas los mayores valores de concentración del Profoxidim.

Manifestando que las muestra 1 y 7 presentan valores menores que las muestras 2, 3, 4, 5 y 6 , la muestra 1 corresponde al Río Paraguay, y la 7 descarga del Tatané; son puntos de muestreos fuera de la superficie cultivada, exceptuando la muestra 7 la cual se encuentra dentro de los límites. Las restantes muestras son extraídas dentro de la arrocera, presentando un valor que no sobrepasa el LMP.

Tabla $N^{\circ} 5$, funda que en la fecha 27/02/2010 (toma de la primera muestra), presenta el mayor valor diferenciándose de las demás fechas, arrojando valores por debajo del LMP.

Es importante el monitoreo en las diferentes etapas del cultivo, para conocer el comportamiento de defensivos agrícolas en el medio ambiente, en razón a las características especificas de cada producto (Peixoto, 2007). Donadío et al., (2009), manifiesta que la Legislación Argentina es más exigente sobre el LMP que los recomendados por el CODEX ALIMENTARIUS y declara que la presencia de niveles excedidos a los LMP es motivo de rechazo de los productos.
Tabla No 4: Resultados Profoxidim. Test de Tukey $(p<0,05)$.

\begin{tabular}{ccccc}
\hline Muestra & Medias & n & EE & \\
\hline 1 & 0,00 & 1 & $1,03 \mathrm{E}-03$ & A \\
7 & $2.0 \mathrm{E}-03$ & 2 & $8,9 \mathrm{E}-0,4$ & A \\
4 & $2,5 \mathrm{E}-03$ & 4 & $6,3 \mathrm{E}-04$ & $\mathrm{~A} \mathrm{~B}$ \\
6 & $3,0 \mathrm{E}-03$ & 2 & $8,9 \mathrm{E}-04$ & A B \\
5 & $4,0 \mathrm{E}-03$ & 2 & $8,9 \mathrm{E}-04$ & $\mathrm{~A} \mathrm{~B}$ \\
2 & $4,0 \mathrm{E}-03$ & 2 & $8,9 \mathrm{E}-04$ & A B \\
3 & 0,01 & 1 & $1,3 \mathrm{E}-03$ & B \\
\hline
\end{tabular}

Tabla No 5: Resultados de Cyhalofop Butil. Test de Tukey $(p<0,05)$.

\begin{tabular}{ccccc}
\hline Fecha & Medias & $n$ & & \\
\hline $19 / 12 / 2011$ & $2.9 E-04$ & 7 & $5.2 E-04$ & $A$ \\
$15 / 04 / 2011$ & $3.3 E-04$ & 6 & $5.6 E-04$ & $A$ \\
$17 / 02 / 2012$ & $5.7 E-04$ & 7 & $5.2 E-04$ & $A$ \\
$11 / 10 / 2010$ & $5.7 E-04$ & 7 & $5.2 E-04$ & $A$ \\
$09 / 10 / 2011$ & $1.7 E-03$ & 6 & $5.6 E-04$ & $A$ \\
$24 / 11 / 2010$ & $1.9 E-03$ & 7 & $5.2 E-04$ & $A$ \\
$10 / 02 / 2011$ & $2.3 E-03$ & 7 & $5.2 E-04$ & $A$ \\
$27 / 02 / 2010$ & 0.01 & 8 & $4.9 E-04$ & $B$ \\
\hline
\end{tabular}




\section{CONCLUSIONES}

En base a los parámetros vigentes establecidos; es posible afirmar que ningún principio activo de los agroquímicos analizados en agua, suelo, sedimento y grano sobrepasan los límites máximos permisibles.

En las muestras de agua, sedimento, suelo y grano, analizadas por fechas de muestreo no presentan relevancia.

Las muestras analizadas por lugar de muestreo establecen con certeza que fuera de la zona de cultivo, toma del Río Paraguay (San Carlos) y aguas arriba del arroyo Caracol (Cancha Larga) no están presentes los defensivos agrícolas analizados. Es decir que las muestras $\mathrm{N}^{\circ} 1$ y $\mathrm{N}^{\circ} 7$ no presentan valores detectables y las restantes muestras presentan valores por debajo del LMP.

\section{BIBLIOGRAFIA}

Arregui, M.; Puricelli, E. 2008. Mecanismo de acción de plagicidas. $1^{\circ}$ edición .ISBN:978-987-05-4191-2. CDD: 661060 .Impreso en Argentona

Donadío de Gandolfi, M.; García, S; Ghersa, CM; Haas, A; Larripa, I; Marra, CA; Ricca, A; Ronco, A; Villamillaamil Lepori, E. 2009. Evaluación de la información científica vinculada al Glifosato en su incidencia sobre la salud humana y el ambiente Consejo Nacional de Investigación Científica Y Técnicas- (CONICET)- Comisión Nacional de investigación sobre agroquímicos decreto 21/ 2009.
European Commission 178/2002: Principios y Requisitos Generales de la Legislación Alimenticia Considerando 12 Vigencia 2005.

Fasola M.; Ruiz, X. 1996. The value of rice fields as subtitutes for natural wetlands for waterbirds in the Mediterranean Region. Colonial waterbirds 19 (Special Pub. 1): 122-128.

Moulin, JF; Iriarte, L; Brieva, S. 2011. Escenarios Futuros para el arroz argentino en el mundo. VII Congreso Brasileiro de Arroz Irrigado, Balneario Camboriú, SC, Brasil. ANAIS, V N 1.

Peixoto, SC. 2007, Estudo da estabilidade a campo dos pesticidas Carbofurano e Quinclorane em água de lavoura de arroz irrigado empregando SPE e HPLC-DAD. 122f. Dissertação (Mestredo em Química)- Universidade Federal de Santa María, RS, Brasil.

Ramsar- xx31-26 de febrero 2010. www.ramsar.org/pdf/ wwd/10/wwd2010_aa_leaflet_s.pdf.

Teló, Marchesan, Oliveira, Zanella, Avila, Perira. 2011. Residuos de agrotóxicos em agua de irrigaç āo do arroz irrigado. VII Congreso Brasileiro de Arroz Irrigado, Balneario Camboriú, SC, Brasil. ANAIS, V Nº2. 